\title{
Relation Between Respiratory Muscle Strength and Skeletal Muscle Mass and Hand Grip Strength in the Healthy Elderly
}

\author{
Hyun iee Shin, MD, Don-Kyu Kim, MD, PhD, Kyung Mook Seo, MD, PhD, \\ Si Hyun Kang, MD, PhD, Sang Yoon Lee, MD, PhD, Sunhan Son, MD
}

Department of Physical Medicine and Rehabilitation, Chung-Ang University College of Medicine, Seoul, Korea

\begin{abstract}
Objective To evaluate sarcopenic indices in relation to respiratory muscle strength (RMS) in elderly people. Methods This study included 65 volunteers over the age of 60 (30 men and 35 women). The skeletal muscle mass index (SMI) was measured using bioimpedance analysis. Limb muscle function was assessed by handgrip strength (HGS), the Short Physical Performance Battery (SPPB), and gait speed. RMS was addressed by maximal inspiratory pressure (MIP) and maximal expiratory pressure (MEP) using a spirometer. The relationships between RMS and other sarcopenic indices were investigated using the Pearson correlation coefficients and multiple regression analysis adjusted for age, HGS, and SPPB.

Results Both MIP and MEP were positively correlated with SMI ( $\mathrm{r}=0.451$ and $\mathrm{r}=0.388$, respectively, $\mathrm{p}<0.05$ in both). HGS showed a significant correlation with both MIP and MEP $(r=0.560, p<0.01$ and $r=0.393, p<0.05$, respectively). There was no significant correlation between gait speed and either MIP or MEP. The SPPB was positively correlated with MEP $(\mathrm{r}=0.436, \mathrm{p}<0.05)$. In the multiple regression analysis, MIP was significantly associated with HGS and SMI $(\mathrm{p}<0.001$ and $\mathrm{p}<0.05$, respectively), while MEP was related only with HGS $(\mathrm{p}<0.05)$.

Conclusion This study suggests that respiratory muscles, especially inspiratory muscles, are significantly related to limb muscle strength and skeletal muscle mass. The clinical significance of MIP and MEP should be further investigated with prospective studies.
\end{abstract}

Keywords Sarcopenia, Respiratory muscles, Muscle strength, Skeletal muscle, Spirometry

Received August 2, 2016; Accepted November 1, 2016

Corresponding author: Don-Kyu Kim

Department of Physical Medicine and Rehabilitation, Chung-Ang University Hospital, Chung-Ang University College of Medicine, 102 Heukseok-ro, Dongjak-gu, Seoul 06973, Korea. Tel: +82-2-6299-1881, Fax: +82-2-6298-1866, E-mail: donkim21@cau.ac.kr

ORCID: Hyun iee Shin (http://orcid.org/0000-0002-9748-7585); Don-Kyu Kim (http://orcid.org/0000-0001-8619-830X); Kyung Mook Seo (http://orcid. org/0000-0002-9762-085X); Si Hyun Kang (http://orcid.org/0000-0003-0205-067X); Sang Yoon Lee (http://orcid.org/0000-0002-2906-3094); Seon Hahn Sohn (http://orcid.org/0000-0003-2158-5880).

@ This is an open-access article distributed under the terms of the Creative Commons Attribution Non-Commercial License (http://creativecommons.org/ licenses/by-nc/4.0) which permits unrestricted noncommercial use, distribution, and reproduction in any medium, provided the original work is properly cited. Copyright $\odot 2017$ by Korean Academy of Rehabilitation Medicine 


\section{INTRODUCTION}

Sarcopenia is an age-related phenomenon characterized by the generalized loss of skeletal muscle mass [1]. On average, people lose approximately $30 \%$ of their muscle mass between the ages of 20 and 80 [2]. In the elderly, however, sarcopenia results in reduced mobility and functional impairment $[2,3]$. It has therefore it has become critical to establish the exact criteria of sarcopenia assuming the crucial consequences.

The current consensus definition of sarcopenia $[4,5]$ is based on low muscle mass and low muscle function according to the European Working Group on Sarcopenia in Older People (EWGSOP) [4]. Both muscle strength and physical performance should be assessed to evaluate muscle function. Muscle strength can be measured using hand grip strength (HGS), which is also a good predictor of clinical outcomes in those with lower extremity muscle weakness [6]. Physical performance can be assessed by the Short Physical Performance Battery (SPPB) and gait speed. According to the International Working Group on Sarcopenia (IWGS), sarcopenia is defined by low muscle mass and poor physical function, e.g., gait speed [5] While the exact definition and criteria for diagnosing sarcopenia are still in dispute, the major criteria currently focus on skeletal muscle mass and extremity muscle strength or function.

There have been recent attempts to estimate the risk of sarcopenia affecting the respiratory muscles [6-9]. Because sarcopenia is a generalized process, it is believed to involve both the limb muscles and the respiratory muscles. Deterioration of respiratory muscle strength can result in significant clinical outcomes. Impaired ventilation results in decreased oxygenation and multisystem organ dysfunction secondary to hypoxia. It is widely believed that impaired ventilation may also contribute to an increased risk of cardiovascular disease through the combination of decreased exercise capacity and higher oxidative stress [10]. In addition, a decline in respiratory muscle strength impedes mobility, which is associated with increased disability, institutionalization, and a worse quality of life in the elderly [3]. Hence, it is critical to evaluate how sarcopenia affects the respiratory muscles along with physical performance and skeletal muscle strength, and how all of these factors correlate in the elderly.

This study aims to evaluate skeletal muscle mass, skele- tal muscle strength, and physical performance in relation to respiratory muscle strength measured by maximum inspiratory pressure (MIP) and maximal expiratory pressure (MEP) in the Korean elderly.

\section{MATERIALS AND METHODS}

\section{Participants}

The study group was composed of healthy volunteers (30 men and 35 women) aged 60 and older. The participants were recruited through advertisements, and the purpose and nature of the study were explained on the written consent form. Participants with a history of respiratory disease (such as chronic obstructive pulmonary disease, lung cancer, previous lung surgery, chest wall deformity, or a current diagnosis of tuberculosis) and a history of major joint surgery were excluded. Those with severe osteoarthritis were also excluded, as this could affect limb muscle function. The study protocol was approved by the Chung-Ang University Hospital Institutional Review Board (C2015141 (1599)).

\section{Assessment of skeletal muscle mass}

Skeletal muscle mass was assessed using bioelectrical impedance analysis (BIA), InBody S10 (Biospace, Seoul, Korea). BIA is a non-invasive, convenient tool that has minimal variation between observers. There was a study that proved a strong correlation with skeletal muscle mass measured with dual-energy X-ray absorptiometry (DXA) and measurement of lean body mass using BIA [11]. Another study successfully demonstrated a strong correlation between MRI-measured and BIA-measured skeletal muscle mass [12]. However, BIA is known to be inaccurate at extremes of the BMI range or in the severely ill [13]. There are also multiple factors that may influence the reliability of BIA measurements, including the position of the electrodes and the patient's body position. To improve the reliability, only one technician operated the instrument in this study. Participants were asked not to eat or exercise for at least 8 hours and to urinate prior to the measurements. After a subject's height and weight were measured, four electrodes were attached to the extremities in the supine position [14]. Skeletal muscle mass (SMM) was obtained through body composition analysis. The skeletal muscle mass index (SMI) [15] was calculated as SMI $(\%)=\operatorname{SMM}(\mathrm{kg}) /$ weight $(\mathrm{kg}) \times 100$. 
Measurements of limb muscle strength

Upper extremity strength was evaluated by HGS. HGS was measured using a hand-grip dynamometer, T.K.K.5401 (Takei Scientific Instruments, Tokyo, Japan), which has been widely used in clinical studies $[16,17]$. Participants were asked to sit in a straight-backed chair with their feet flat on the floor, adduct and neutrally rotate the shoulder, flex the elbow to $90^{\circ}$, and place the forearm in a neutral position with the wrist between $0^{\circ}$ and $30^{\circ}$ of extension and between $0^{\circ}$ and $15^{\circ}$ of ulnar deviation. The arm was not supported, and a hand-grip dynamometer was presented in line with the forearm. Participants were instructed to squeeze the handle as hard as possible for 3 seconds, and the maximum contractile force $(\mathrm{kg})$ was recorded. The tests were performed three times on both hands, alternating between the right and left hands with a 60 -second rest period between each trial [18]. The highest value among the three repeated measurements was used for analysis.

\section{Measurements of physical performance}

To assess physical performance, especially that of the lower extremities, the SPPB was employed. The SPPB is a simple test to measure lower extremity function using tasks that mimic daily activities [19]. The SPPB examines 3 areas of lower extremity function: static balance, gait speed, and endurance. Static balance was assessed by the ability to stand with the feet together in side-by-side, semi-tandem, and tandem positions. Gait speed was assessed by measuring the time taken to walk 8 feet at one's usual pace. Endurance was measured by the time taken to rise from a chair and return to the seated position 5 times.

\section{Measurements of respiratory muscle strength}

To acknowledge muscle strength that are independent of the condition of the lung, the most widely applied technique in the assessment of inspiratory and expiratory muscle strength, MIP and MEP were measured using a digital spirometer (Pony FX; COSMED, Rome, Italy) [20]. MIP and MEP are widely used to evaluate global inspiratory and expiratory muscle strength and measure the maximum pressures at the mouth $[21,22]$. Clinically, MIP is the most common technique in the assessment of inspiratory muscle strength, and MEP is the only test generally available for measuring expiratory muscle strength [20]. The portable spirometer used in this study is accurate and reliable when compared to formal laboratory-based spirometry [23]. The results, however, are highly dependent on subject motivation, and there might be leaks around the mouth during the performance, especially at higher pressures and in older subjects [20]. To minimize these errors, at least 5 trials of spirometry were undertaken, and the participants were asked to take a deep breath and securely seal their lips around the mouthpiece to prevent air leakage. The measurements were carried out in the seated position with participants wearing a nose clip. The subjects were encouraged to maximally inspire for MIP and maximally expire for MEP. Between each of the 5 trials, there was a 1-minute rest period. The maximum values of each maneuver were recorded for analysis [21].

\section{Statistical analysis}

The demographic characteristics and measurements of the participants as well as all numerical variables are expressed as mean \pm standard deviation. Correlations between outcome variables were analyzed with the Pearson correlation test. Multiple regression analysis was used to identify independent factors predicting each person's respiratory muscle strength, adjusting for age, SMI, hand grip strength, and SPPB. Significance was defined by $\mathrm{p}$ values less than 0.05 . All data were statistically analyzed using the Statistical Package for Social Sciences for Windows ver. 17.0 (SPSS Inc., Chicago, IL, USA).

\section{RESULTS}

\section{Characteristics of subjects}

A total of 33 male subjects and 35 female subjects were recruited, however, 3 participants ( 2 with chronic obstructive pulmonary disease and 1 with an amputated index finger) were excluded. The final study population consisted of 65 subjects ( 30 men and 35 women). The baseline characteristics of the subjects are presented in Table 1 . The mean age of the participants was $69.90 \pm 7.63$ (Table 1).

Correlation between respiratory muscle strength and SMM, HGS, and physical performance indices

Both MIP and MEP were positively correlated with SMI ( $r=0.451$ and $r=0.388$, respectively, $p<0.05$ in both) 
(Table 2). HGS also showed significant correlations with both MIP and MEP ( $r=0.560, p<0.01$ and $r=0.393, p<0.05$, respectively) (Table 2). Gait speed was not significantly correlated with either MIP or MEP, however, SPPB was positively correlated with MEP $(\mathrm{r}=0.436, \mathrm{p}<0.05)$ (Table 2$)$.

Table 1. Characteristics of subjects

\begin{tabular}{|cc}
\hline \multicolumn{1}{c}{ Characteristic } & Value \\
\hline Age $(\mathrm{yr})$ & $69.90 \pm 7.63$ \\
\hline Gender & 30 \\
\hline Male & 35 \\
\hline Female & $159.47 \pm 5.65$ \\
\hline Height $(\mathrm{cm})$ & $62.58 \pm 6.80$ \\
Weight $(\mathrm{kg})$ & $24.58 \pm 2.69$ \\
\hline Body mass index $\left(\mathrm{kg} / \mathrm{m}^{2}\right)$ & $43.46 \pm 6.90$ \\
Skeletal mass index $(\%)$ & $26.57 \pm 5.90$ \\
\hline Hand grip strength $(\mathrm{kg})$ & $76.04 \pm 28.26$ \\
\hline MIP $\left(\mathrm{cmH} \mathrm{c}_{2} \mathrm{O}\right)$ & $82.06 \pm 23.15$ \\
\hline MEP $\left(\mathrm{cmH} \mathrm{H}_{2} \mathrm{O}\right)$ & $0.99 \pm 0.25$ \\
\hline Gait speed $(\mathrm{m} / \mathrm{s})$ & $10.24 \pm 1.53$ \\
\hline SPPB & \\
\hline
\end{tabular}

Values are presented as mean \pm standard deviation or number.

MIP, maximal inspiratory pressure; MEP, maximal expiratory pressure; SPPB, short physical performance battery.
Multiple linear regression analysis between respiratory muscle strength and conventional sarcopenic indices

In a multiple linear regression analysis where MIP was a dependent variable, HGS and SMI were significantly related when adjusted for age and SPPB $(\beta=1.876, \mathrm{p}<0.001$ and $\beta=1.964, p=0.01$, respectively) (Table 3). In a multiple regression analysis of MEP, however, only hand grip strength was significant $(\beta=1.102, p=0.02)$ (Table 3$)$.

\section{DISCUSSION}

The results of this study demonstrate that there is a positive relationship between respiratory muscle strength and the conventional sarcopenic indices in the Korean elderly. Even after adjusting for other factors, MIP and MEP were both significantly associated with HGS (Table 3 ). The results coincide with previous studies done on respiratory muscle strength and limb muscle strength on other ethnic groups that respiratory muscles clearly have significant relationship with limb muscle strength $[6,24]$. One should note, however, that the Pearson correlation coefficients ( $\mathrm{r}$ ) of MIP with SMI and HGS ( $\mathrm{r}=0.451$, $\mathrm{p}<0.05$ and $\mathrm{r}=0.560, \mathrm{p}<0.01$, respectively) are relatively higher than those with MEP ( $\mathrm{r}=0.388, \mathrm{p}<0.05$ and $\mathrm{r}=0.393$, $\mathrm{p}<0.05$, respectively) (Table 2). Moreover, multiple linear regression analysis shows that SMM, the major factor in diagnosing sarcopenia $[4,5]$, is only significantly associ-

Table 2. Pearson correlation coefficients ( $r$ ) of respiratory muscle strength, limb strength, and skeletal muscle mass

\begin{tabular}{lcccl}
\hline & SMI & HGS & Gait speed & SPPB \\
\hline MIP** $^{* *}$ & $0.451^{*}$ & $0.560^{* *}$ & $0.266^{* *}$ & $0.313^{* *}$ \\
MEP* $^{* *}$ & $0.388^{*}$ & $0.393^{*}$ & $0.219^{* *}$ & $0.436^{*}$ \\
\hline
\end{tabular}

SMI, skeletal muscle mass index; HGS, hand grip strength; SPPB, short physical performance battery; MIP, maximal inspiratory pressure; MEP, maximal expiratory pressure.

${ }^{*} \mathrm{p}<0.05,{ }^{* *} \mathrm{p}<0.01$.

Table 3. Multiple linear regression analysis for respiratory muscle strength using various sarcopenic indices

\begin{tabular}{|c|c|c|c|c|c|c|}
\hline & \multicolumn{3}{|c|}{ MIP } & \multicolumn{3}{|c|}{ MEP } \\
\hline & SE & $\beta$ & p-value & SE & $\beta$ & p-value \\
\hline Age & 0.569 & 0.216 & 0.71 & 0.464 & 1.459 & 0.41 \\
\hline HGS & 23.930 & 1.876 & $<0.001^{* * *}$ & 52.623 & 1.102 & $0.02^{*}$ \\
\hline SMI & 12.785 & 1.964 & $0.01^{*}$ & 0.659 & 0.637 & 0.34 \\
\hline SPPB & 16.536 & 32.685 & 0.06 & 13.615 & 12.441 & 0.37 \\
\hline
\end{tabular}

MIP, maximal inspiratory pressure; MEP, maximal expiratory pressure; HGS, hand grip strength; SMI, skeletal muscle mass index; SE, standard error; $\beta$, standardized coefficient.

${ }^{*} \mathrm{p}<0.05,{ }^{* *} \mathrm{p}<0.01,{ }^{* * *} \mathrm{p}<0.001$. 
ated with MIP and not MEP. These observations may suggest that there is sarcopenic involvement of the respiratory muscles, especially the inspiratory muscles.

Pulmonary function is believed to partially account for the association between general muscle strength and mortality [25]. A sedentary lifestyle combined with deterioration of pulmonary muscle strength due to aging results in a decline in mobility, further accelerating reductions in respiratory muscle strength [26]. There was also a report stating that respiratory muscle strength is associated with a decline in mobility in older persons, independent of limb muscle strength and physical activity [3]. Assuming the significant clinical consequences, there have been many attempts to link respiratory muscle strength and age-related sarcopenia. Recent studies have examined pulmonary function and sarcopenia in the elderly $[8,9]$ using data from a Korean National Health and Nutrition Examination Survey. These studies showed relationships between the forced expiratory volume in 1 second (FEV1) and the forced vital capacity (FVC) with SMM. However, it is assumed that the FEV1 and the FVC rather show the overall function of a lung or pulmonary condition by the compliance of the lung-thorax system, demonstrating the resistance of the airways [27]. The fact that the FEV1 and the FVC values are diagnostic criteria of chronic obstructive pulmonary disease (COPD) proves that they reflect pulmonary function rather than respiratory muscle strength. Moreover, there are studies that suggest that respiratory muscle training has proven effects on MIP and MEP [25,26].

A study looking at 65 Brazilian elderly people demonstrated a positive correlation between lower limb muscle function and respiratory muscle strength by measuring knee flexor/extensor muscle strength, 6-minute walking speed, and MIP/MEP [24]. There was also a study of 62 male nursing home residents in Turkey that suggested MIP is significantly related to HGS independent of other factors [6], which is perfectly in line with the results of our study. These prior studies have demonstrated a positive relationship between respiratory muscle strength and sarcopenia by linking limb muscle function with respiratory muscle strength. None of these studies, however, reported a direct relationship between SMM and respiratory muscle strength. As mentioned above, the current consensus on the diagnosis of sarcopenia is based on low muscle mass and/or variably measured low muscle strength or physical performance [4,5]. Our study shows its value in that it compared the actual SMM with respiratory muscle strength, not only with limb muscle function. To the best of our knowledge, this is the first study to demonstrate an association between SMM and MIP and MEP in the elderly Korean population.

The results of this study are also in conjunction with our previous study about the relationship between respiratory muscle strength and conventional sarcopenic indices in the young Korean population [28], in that respiratory muscle strength, markedly MIP, and various sarcopenic indices are inter-related, which is due to sarcopenia. In our previous study, MIP was the only value associated with SMM and limb muscle function. In this study, both MIP and MEP were independently associated with limb muscle strength. SMM, the most important factor for diagnosing sarcopenia, was only independently associated with MIP. Because of age-related muscle deterioration, we assumed that unlike in the young population, MEP would also have a significant association with skeletal muscle strength, although to a lesser degree than MIP, suggesting that inspiratory muscles are more vulnerable to the age-related changes of sarcopenia. With older population group than our study population, we expect that even the SMM might also be significantly associated with MEP. Future studies with older participants may also be necessary.

It is still debatable whether MIP or MEP is more reliable in reflecting muscle mass in the aging population. Several studies $[29,30]$ have suggested that the expiratory muscles might be more vulnerable to the aging process than the inspiratory muscles. In a study conducted on children with neuromuscular disease, MIP is relatively preserved in these patients, therefore, MEP has been emphasized as a sensitive marker of the respiratory muscle deterioration in these patients [31]. However, in the study with traumatic SCI patients, it was suggested that MEP might not be a more sensitive marker of the respiratory muscle deterioration. In patients with a traumatic SCI, both the inspiratory and expiratory dysfunction developed simultaneously [32]. Moreover, in the study with healthy elderly, there was a significant association between HGS and MIP, but not with MEP, concluding that in sarcopenia, the inspiratory dysfunction may develop earlier than the expiratory dysfunction [6]. In the animal study, there were obvious sarcopenic changes in the 
diaphragm muscles in aging mice than younger group [33]. The diaphragm is a substantial inspiratory muscle, further supporting sarcopenic involvement in inspiratory muscles.

Examining the relationship between respiratory muscle strength and the parameters of physical performance, the SPPB showed a moderate correlation with only MEP $(\mathrm{r}=0.436, \mathrm{p}<0.05)$. The SPPB, however, failed to show significance with MEP in regression analysis (Table 3), indicating that there is no causal relationship between MEP and the SPPB. Based on these results, it is possible that the significant correlation between MEP and the SPPB is due to confounding factors such as age. In addition, despite an average age of around 70, we recruited only healthy individuals with good physical performance, which might have skewed the results. To accomplish the SPPB, the patients had to perform various tasks requiring rather complicated instructions and greater effort in geriatric subjects, which might also explain the results. The significance of MEP in evaluating the physical outcomes of sarcopenia in elderly people, however, merits further investigation. It would also be useful to examine the association between physical performance and the parameters of pulmonary function, such as FEVl or FVC.

This study has some limitations. Assessing the participants' muscle strength and physical function required each participant's maximal voluntary effort. Although we provided a thorough explanation before each trial and each trial was conducted at least 3 times, it is still possible that the results do not reflect the participants' maximal capacity. More importantly, the relatively small sample size may have masked some statistical results.

To date, this is the first study demonstrating an independent relationship between respiratory muscle strength and SMM in the Korean elderly. The fact that sarcopenia is also associated with respiratory muscle strength is crucial, as several rehabilitative exercises and interventions have proven beneficial to strengthening ventilatory muscles $[26,29]$. Loss of respiratory muscle mass and function could be prevented by aptly applying these exercises. Further studies on sarcopenia and its effects on respiratory muscle strength are necessary to improve the life expectancy and quality of life of the geriatric population.

\section{CONFLICT OF INTEREST}

No potential conflict of interest relevant to this article was reported.

\section{REFERENCES}

1. Doherty TJ. Invited review: Aging and sarcopenia. J Appl Physiol (1985) 2003;95:1717-27.

2. Bahat G, Tufan A, Tufan F, Kilic C, Akpinar TS, Kose M, et al. Cut-off points to identify sarcopenia according to European Working Group on Sarcopenia in Older People (EWGSOP) definition. Clin Nutr 2016;35:1557-63.

3. Buchman AS, Boyle PA, Wilson RS, Leurgans S, Shah $\mathrm{RC}$, Bennett DA. Respiratory muscle strength predicts decline in mobility in older persons. Neuroepidemiology 2008;31:174-80.

4. Cruz-Jentoft AJ, Baeyens JP, Bauer JM, Boirie Y, Cederholm T, Landi F, et al. Sarcopenia: European consensus on definition and diagnosis: Report of the European Working Group on Sarcopenia in Older People. Age Ageing 2010;39:412-23.

5. Fielding RA, Vellas B, Evans WJ, Bhasin S, Morley JE, Newman AB, et al. Sarcopenia: an undiagnosed condition in older adults. Current consensus definition: prevalence, etiology, and consequences. International working group on sarcopenia. J Am Med Dir Assoc 2011;12:249-56.

6. Bahat G, Tufan A, Ozkaya H, Tufan F, Akpinar TS, Akin $\mathrm{S}$, et al. Relation between hand grip strength, respiratory muscle strength and spirometric measures in male nursing home residents. Aging Male 2014;17: 136-40.

7. Simoes LA, Dias JM, Marinho KC, Pinto CL, Britto RR. Relationship between functional capacity assessed by walking test and respiratory and lower limb muscle function in community-dwelling elders. Rev Bras Fisioter 2010;14:24-30.

8. Jeon YK, Shin MJ, Kim MH, Mok JH, Kim SS, Kim $\mathrm{BH}$, et al. Low pulmonary function is related with a high risk of sarcopenia in community-dwelling older adults: the Korea National Health and Nutrition Examination Survey (KNHANES) 2008-2011. Osteoporos Int 2015;26:2423-9.

9. Moon JH, Kong MH, Kim HJ. Implication of sarcopenia and sarcopenic obesity on lung function in healthy 
elderly: using Korean National Health and Nutrition Examination Survey. J Korean Med Sci 2015;30:1682-8.

10. Drost EM, Skwarski KM, Sauleda J, Soler N, Roca J, Agusti A, et al. Oxidative stress and airway inflammation in severe exacerbations of COPD. Thorax 2005;60: 293-300.

11. Heymsfield SB, Gallagher D, Grammes J, Nunez C, Wang Z, Pietrobelli A. Upper extremity skeletal muscle mass: potential of measurement with single frequency bioimpedance analysis. Appl Radiat Isot 1998;49:4734.

12. Janssen I, Heymsfield SB, Baumgartner RN, Ross R. Estimation of skeletal muscle mass by bioelectrical impedance analysis. J Appl Physiol (1985) 2000;89: 465-71.

13. Kyle UG, Bosaeus I, De Lorenzo AD, Deurenberg P, Elia M, Manuel Gomez J, et al. Bioelectrical impedance analysis. Part II. Utilization in clinical practice. Clin Nutr 2004;23:1430-53.

14. Sergi G, De Rui M, Stubbs B, Veronese N, Manzato E. Measurement of lean body mass using bioelectrical impedance analysis: a consideration of the pros and cons. Aging Clin Exp Res 2016 Aug 27 [Epub]. https:// doi.org/10.1007\%2Fs40520-016-0622-6.

15. Janssen I, Heymsfield SB, Ross R. Low relative skeletal muscle mass (sarcopenia) in older persons is associated with functional impairment and physical disability. J Am Geriatr Soc 2002;50:889-96.

16. Arai H, Akishita M, Chen LK. Growing research on sarcopenia in Asia. Geriatr Gerontol Int 2014;14 Suppl 1:1-7.

17. Pedrero-Chamizo R, Albers U, Tobaruela JL, Melendez A, Castillo MJ, Gonzalez-Gross M. Physical strength is associated with Mini-Mental State Examination scores in Spanish institutionalized elderly. Geriatr Gerontol Int 2013;13:1026-34.

18. Innes EV. Handgrip strength testing: a review of the literature. Aust Occup Ther J 1999;46:120-40.

19. Guralnik JM, Ferrucci L, Simonsick EM, Salive ME, Wallace RB. Lower-extremity function in persons over the age of 70 years as a predictor of subsequent disability. N Engl J Med 1995;332:556-61.

20. Syabbalo N. Assessment of respiratory muscle function and strength. Postgrad Med J 1998;74:208-15.

21. American Thoracic Society/European Respiratory So- ciety. ATS/ERS Statement on respiratory muscle testing. Am J Respir Crit Care Med 2002;166:518-624.

22. Lee J, Hwang S, Han S, Han D. Effects of stretching the scalene muscles on slow vital capacity. J Phys Ther Sci 2016;28:1825-8.

23. Bambra G, Jalota L, Kapoor C, Mills PK, Vempilly JJ, Jain VV. Office spirometry correlates with laboratory spirometry in patients with symptomatic asthma and COPD. Clin Respir J 2015 Dec 1 [Epub]. https://doi.org/ 10.1111\%2Fcrj.12419.

24. Giua R, Pedone C, Scarlata S, Carrozzo I, Rossi FF, Valiani V, et al. Relationship between respiratory muscle strength and physical performance in elderly hospitalized patients. Rejuvenation Res 2014;17:366-71.

25. Buchman AS, Boyle PA, Wilson RS, Gu L, Bienias JL, Bennett DA. Pulmonary function, muscle strength and mortality in old age. Mech Ageing Dev 2008;129: 625-31.

26. Kim J, Davenport P, Sapienza C. Effect of expiratory muscle strength training on elderly cough function. Arch Gerontol Geriatr 2009;48:361-6.

27. Neder JA, Andreoni S, Lerario MC, Nery LE. Reference values for lung function tests. II. Maximal respiratory pressures and voluntary ventilation. Braz J Med Biol Res 1999;32:719-27.

28. Ro HJ, Kim DK, Lee SY, Seo KM, Kang SH, Suh HC. Relationship between respiratory muscle strength and conventional sarcopenic indices in young adults: a preliminary study. Ann Rehabil Med 2015;39:880-7.

29. Kim J, Sapienza CM. Implications of expiratory muscle strength training for rehabilitation of the elderly: Tutorial. J Rehabil Res Dev 2005;42:211-24.

30. Mizuno M. Human respiratory muscles: fibre morphology and capillary supply. Eur Respir J 1991;4:587601.

31. Schramm CM. Current concepts of respiratory complications of neuromuscular disease in children. Curr Opin Pediatr 2000;12:203-7.

32. Kang SW, Shin JC, Park CI, Moon JH, Rha DW, Cho DH. Relationship between inspiratory muscle strength and cough capacity in cervical spinal cord injured patients. Spinal Cord 2006;44:242-8.

33. Greising SM, Mantilla CB, Gorman BA, Ermilov LG, Sieck GC. Diaphragm muscle sarcopenia in aging mice. Exp Gerontol 2013;48:881-7. 